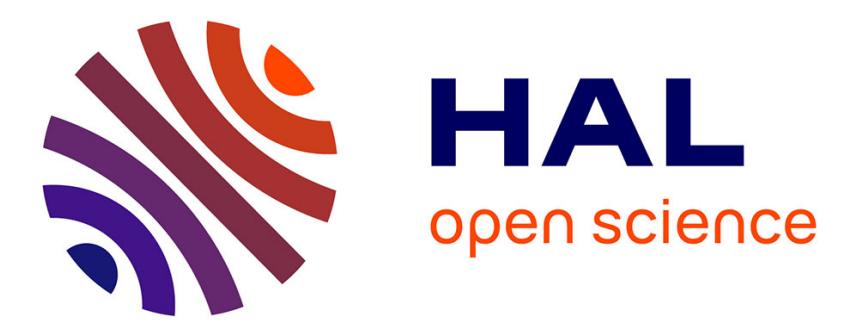

\title{
Dry snow backscattering sensivity on density change for SWE estimation
}

\author{
Nikola Besic, Gabriel Vasile, Jocelyn Chanussot, Srdjan Stankovic, \\ Jean-Pierre Dedieu, Guy d'Urso, Dider Boldo, Jean-Philippe Ovarlez
}

\section{- To cite this version:}

Nikola Besic, Gabriel Vasile, Jocelyn Chanussot, Srdjan Stankovic, Jean-Pierre Dedieu, et al.. Dry snow backscattering sensivity on density change for SWE estimation. IGARSS 2012 - IEEE International Geoscience and Remote Sensing Symposium, Jul 2012, Munich, Germany. pp.4418-4421, 10.1109/IGARSS.2012.6350393 . hal-00726925v2

\section{HAL Id: hal-00726925 \\ https://hal.science/hal-00726925v2}

Submitted on 31 Aug 2012

HAL is a multi-disciplinary open access archive for the deposit and dissemination of scientific research documents, whether they are published or not. The documents may come from teaching and research institutions in France or abroad, or from public or private research centers.
L'archive ouverte pluridisciplinaire HAL, est destinée au dépôt et à la diffusion de documents scientifiques de niveau recherche, publiés ou non, émanant des établissements d'enseignement et de recherche français ou étrangers, des laboratoires publics ou privés. 


\title{
DRY SNOW BACKSCATTERING SENSITIVITY ON DENSITY CHANGE FOR SWE ESTIMATION
}

\author{
N. Besic ${ }^{1,2}$, G. Vasile ${ }^{1}$, J. Chanussot ${ }^{1}$, S. Stankovic ${ }^{2}$, J.-P. Dedieu ${ }^{3}$, G. d'Urso ${ }^{4}$, D. Boldo ${ }^{4}$, J.-P. Ovarlez ${ }^{5}$ \\ ${ }^{1}$ GIPSA lab, CNRS/Grenoble-INP, Grenoble, France, nikola.besic@gipsa-lab.grenoble-inp.fr \\ ${ }^{2}$ University of Montenegro, Podgorica, Montenegro, srdjan@ac.me \\ ${ }^{3}$ LTHE, CNRS/Grenoble-INP, Grenoble, France, jean.pierre.dedieu@ujf.grenoble.fr \\ ${ }^{4}$ EDF, R\&D STEP, Chatou, France, guy.durso@edf.fr \\ ${ }^{5}$ ONERA, DEMR/TSI, Palaiseau, France, jean-philippe.ovarlez@onera.fr
}

\begin{abstract}
This paper provides comprehensive analysis of the dry snow pack backscattering coefficient dependence on the density change, for various SAR sensor parameters and chosen dry snow pack parameters, characteristic for the region of French Alps. As the result, qualitative conclusions, based on applying fundamental scattering theories (Rayleigh scattering model, Quasi Crystalline Approximation, Integral Equation Model) on the particular distributed target are presented. They represent the ground for semi-empirical models, which may provide a satisfactory link between backscattering coefficient and snow density (as one of the quantities defining SWE), as well as the guidelines for the further radar acquisitions over the Alpine region in France.
\end{abstract}

Index Terms - SAR, backscattering, SWE, sensitivity, snow density

\section{INTRODUCTION}

Snow water equivalent (SWE) is defined as the depth of the layer of liquid water that would be produced if all the ice in the snow pack were melted [1]. It is function of two independent physical quantities, which characterise the snow pack: density $\left(\rho_{s}\right)$ and $\operatorname{depth}(d)$ :

$$
S W E=\frac{1}{\rho_{w}} \int_{0}^{d} \rho_{s} \mathrm{~d} z
$$

with $\rho_{w}$ as liquid water density. SWE estimation is important due to the wide usage of this kind of water resource in agriculture, power production, as the drinking water supply etc. Due to the numerous difficulties in performing "in situ" measurements (high altitudes, global spatial distribution), the estimation of this quantity turns out to be suitable for SAR remote sensing application [2,3]. In order to be able to deduce SWE, as a physical quantity, from the SAR image, it is

This work was supported by the French National R\&DProgram TSEN-X PEPS UJF-CNRS 2011. necessary to relate both the snow density and the depth to the backscattering coefficient. This may represent a problem, due to the fact that backscattering is a function of many parameters, both sensor (frequency, polarization, angle) and target ones. This paper addresses one particular target parameter snow density.

More concretely, it deals dry snow density, where dry snow can be defined as snow consisted just of the ice crystals embedded in air, without any liquid water. Dry snow dielectric constant (eq.2) is a real number, which indicates the absence of absorption losses. It is a function of dry snow density $\left(\rho_{d s}\right)$, which could be expressed through the ice volume fraction $\left(f_{i c e}\right)$ and the ice dielectric constant $\left(\rho_{i c e}\right)$. Due to the backscattering dependence on target dielectric properties through medium boundaries influence on electromagnetic waves propagation (Fresnel transmission coefficient, snow and underlying ground surface scattering), the dielectric constant appears to be the first link between the snow density and the backscattering coefficient.

$$
\varepsilon_{d s}^{\prime}=1+1.9 \rho_{d s}=1+1.9 \rho_{i c e} f_{i c e}
$$

Aside from this, density also defines snow medium extinction properties $\left(\kappa_{e}\right)$, introduced through radiative transfer equation:

$$
\frac{d I(\vec{r}, \vec{s})}{d s}=-\kappa_{e} I(\vec{r}, \vec{s})+\int_{4 \pi} P\left(\vec{r}, \overrightarrow{s^{\prime}}\right) I\left(\vec{r}, \overrightarrow{s^{\prime}}\right) \mathrm{d} \Omega^{\prime}
$$

which models electromagnetic waves propagation through some particular medium. Change of EM wave intensity depends on the extinction, represented by the first term, and the amount of scattered radiation which is being re-scattered into the direction of interest, described by the second term using phase matrix $P\left(\vec{r}, \overrightarrow{s^{\prime}}\right)$. Extinction losses, generally related to the absorption and the scattering losses, in the case of dry snow refer only to the losses due to the scattering. This can be justified by the lack of liquid water and the ice crystals dielectric properties (purely real dielectric constant). This paper threats two volume scattering types in parallel. First, 
single independent scattering assumption, characteristic for Rayleigh scattering model, which considers that the waves scattered of the particle do not affect other particles scattering. It can be considered as valid as far as the medium density is not significantly big, which makes it suitable for dry snow analysis. Second, multiple scattering assumption, modelled by Quasi Crystalline Approximation (QCA), which doesn't neglect the influence of the scattered waves on other particles scattering. Furthermore, it assumes waves coherence, and it stays valid even in the case of denser media [4].

Two volume scattering types are characterized with two different functions of extinction coefficient against snow density. Therefore, the parallel study turns out to be quite significant for proper understanding of snow cover backscattering mechanism. The important remark would be that the study is based on the ice particles spherical shape assumption, which simplifies extinction derivation. The particles radius is taken as a function of snow sphericity $(s)$ and dendricity $(d)$ [5]:

$$
r_{i c e}=d+(1-d)(4-s)\left[10^{-4} m\right]
$$

The backscattering simulator, described in this paper, and used to draw qualitative conclusions concerning snow density influence on total backscattering in three microwave bands (L, C and $\mathrm{X}$ ), is integrating two previously described links.

\section{BACKSCATTERING MECHANISM}

Total backscattering $\left(\sigma_{0}\right)$ of the snow pack as a distributed target can be decomposed as the sum of the four components $[3,6]$ (fig. 1):

1. Snow pack surface component $\left(\sigma_{s}\right)$;

2. Underlying ground surface component $\left(\sigma_{g r}\right)$;

3. Snow volume component $\left(\sigma_{v}\right)$;

4. Ground-volume interaction component $\left(\sigma_{g v}\right)$;

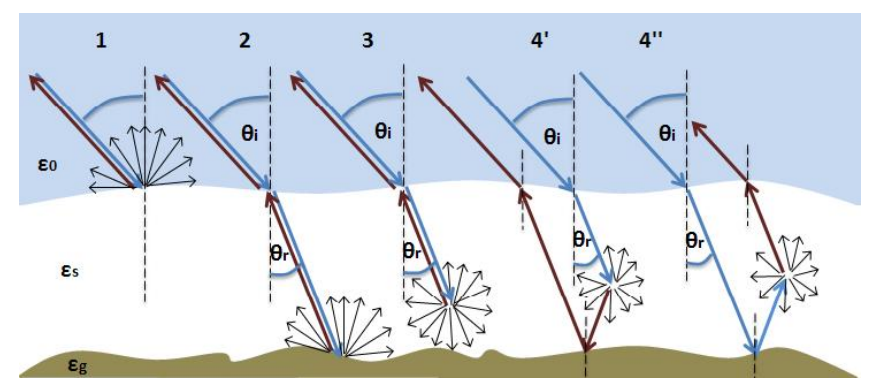

Fig. 1. Snow pack backscattering mechanism

$$
\sigma_{0}=\sigma_{s}+\sigma_{g r}+\sigma_{v}+\sigma_{g v}
$$

For co-polarized channels ( $p p=h h \vee v v$ ) the backscattering components are given as [6]:

$$
\sigma_{s}=\sigma\left(\varepsilon_{0}, \theta_{i}, \theta_{i}, \phi_{s}-\phi, \frac{\varepsilon_{s}}{\varepsilon_{0}}, \sigma_{R M S}^{s}, l_{c}^{s}\right)
$$

$$
\begin{gathered}
\sigma_{g r}=\frac{\mu_{i}}{\mu_{r}} T_{p p}^{2}\left(\theta_{r}, \theta_{i}\right) e^{\left(\frac{-2 \kappa_{e} d}{\mu_{r}}\right)} \sigma\left(\varepsilon_{s}, \theta_{r}, \theta_{r}, \phi_{s}-\phi, \frac{\varepsilon_{g}}{\varepsilon_{s}}, \sigma_{R M S}^{g}, l_{c}^{g}\right) \\
\sigma_{v}=a \frac{\mu_{i}}{2} T_{p p}^{2}\left(\theta_{r}, \theta_{i}\right)\left[1-e^{\left(\frac{-2 \kappa_{e} d}{\mu_{r}}\right)}\right] P_{p p}\left(\mu_{r},-\mu_{r}, \phi_{s}-\phi\right) \\
\sigma_{g v}^{\prime}=\mu_{i} a R_{p p}\left(\theta_{r}, \theta_{i}, \varepsilon_{g}\right) \frac{\kappa_{e} d}{\mu_{r}} e^{\left(\frac{-2 \kappa_{e} d}{\mu_{r}}\right)-4 k^{2} \sigma_{R M S}^{s} \mu_{r}^{2}} \\
\cdot T_{p p}^{2}\left(\theta_{r}, \theta_{i}\right) P_{p p}\left(-\mu_{r},-\mu_{r}, \phi_{s}-\phi\right) \\
\sigma_{g v}^{\prime \prime}=\mu_{i} a R_{p p}\left(\theta_{r}, \theta_{i}, \varepsilon_{g}\right) \frac{\kappa_{e} d}{\mu_{r}} e^{\left(\frac{-2 \kappa_{e} d}{\mu_{r}}\right)-4 k^{2} \sigma_{R M S}^{s} \mu_{r}^{2}} \\
\cdot T_{p p}^{2}\left(\theta_{r}, \theta_{i}\right) P_{p p}\left(\mu_{r}, \mu_{r}, \phi_{s}-\phi\right)
\end{gathered}
$$

Snow pack surface backscattering coefficient (eq.6) is calculated using Integral Equation Model $(\sigma)$ as a function of local incidence elevation angle $\left(\theta_{i}\right)$, azimuth angle $\left(\phi_{s}\right)$, snow dielectric constant, surface root mean square hight $\left(\sigma_{R M S}^{s}\right)$ and its correlation length $\left(l_{c}^{s}\right)$ [6]. The backscattering strongly depends on the incident waves wavelength, which makes it also a function of the incident media dielectric constant $\left(\varepsilon_{0}\right)$.

For better comprehension of underlying ground backscattering component (eq.7), three different impacts on backscattering coefficient can be distinguished:

- Air-snow interface direct impact, represented through the incident and refracted angle $\left(\theta_{r}\right)$, cosines ratio $\left(\frac{\mu_{i}}{\mu_{r}}\right)$ and squared Fresnel transmission coefficient $\left(T_{p p}^{2}\left(\theta_{r}, \theta_{i}\right)\right)$, both affected by the density through the snow dielectric constant.

- Snow medium propagation impact, meaning the extinction $\left(e^{\left(\frac{-2 \kappa_{e} d}{\mu_{r}}\right)}\right)$ given as a direct density function.

- Ground surface backscattering in the presence of snow, derived by applying the same model as in the case of snow surface. The presence of snow, through snow dielectric constant, defines the refracted angle (behaving as an incidence one at the ground surface), refracted wave wavelength and groundsnow dielectric contrast $\left(\frac{\varepsilon_{g}}{\varepsilon_{s}}\right)$.

Volume backscattering component (eq.8) is also affected by air-snow boundary transmission, but importantly, it is dependent on snow density through extinction losses. It is function of normalized (relative to the scattering coefficient) Rayleigh phase matrix $\left(P_{p p}\left(\mu_{r},-\mu_{r}, \phi_{s}-\phi\right)\right)$, giving the amount of energy scattered in the backward direction.

Component named ground-volume interaction is related to the waves directed by the volume and then specularly reflected toward the antenna (eq.9) and the waves specularly reflected of the ground and then redirected toward the direction of interest by the volume (eq.9). Specular reflection is introduced through Fresnel reflection power coefficient $R_{p p}\left(\theta_{r}, \theta_{i}, \varepsilon_{g}\right)$ corrected with a factor $4 k^{2} \sigma_{R M S}^{s} \mu_{r}^{2}$ which brings the terrain roughness into the account. Direction change caused by volume is modelled via normalized Rayleigh phase matrix.

\section{BACKSCATTERING SIMULATOR}

The built simulator is based on the previously described backscattering mechanism. Integral Equation Model (IEM) is 


\begin{tabular}{||l|l||}
\hline Input parameter & Value $(\mathrm{s})$ \\
\hline Snow density & $180,190,200,210,220 \mathrm{~kg} / \mathrm{m}^{3}$ \\
\hline Snow depth & $0.2 \mathrm{~m}$ \\
\hline Frequency & $1.5(\mathrm{~L}), 6(\mathrm{C}), 10(\mathrm{X}) \mathrm{GHz}$ \\
\hline Particle sphericity & 0.5 \\
\hline Particle dendricity & 0.5 \\
\hline Ice dielectric constant & 3.15 \\
\hline Snow RMS height & $10 \mathrm{~mm}$ \\
\hline Snow correlation length & $50 \mathrm{~mm}$ \\
\hline Ground dielectric constant & 6 \\
\hline Ground RMS height & $10 \mathrm{~mm}$ \\
\hline Ground correlation length & $50 \mathrm{~mm}$ \\
\hline
\end{tabular}

Table 1. Simulation input parameters

used for simulating surface backscattering, with the assumption of exponentially correlated surface. Rayleigh scattering model and Quasi Crystalline Approximation are modelling snow volume behaviour.

The output result is the angular distribution of backscattering coefficient for different values of snow density backscattering sensitivity on the density change. Incidence angle values from $0^{\circ}$ up to $70^{\circ}$ are considered.

Input data, chosen according to the available data, provided by EDF (Electricite de France) and according to the regional Meteo France forecasts are given in the table 1.

Surface RMS height represents an exception. Namely, 10 $m m$ ground RMS height doesn't correspond precisely to the actual terrain roughness. The limits of IEM model and the necessity to use the same surface properties for each frequency band caused the choice of a limiting value for $10 \mathrm{GHz}$ frequency.

$$
\frac{2 \pi}{\lambda} \sigma_{R M S}<3
$$

\section{RESULTS}

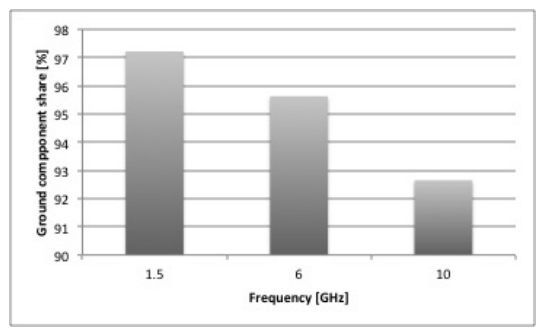

Fig. 2. $\sigma_{g r}$ share in total backscattering

In all three frequency bands, the absolutely most dominant component is the underlying ground one (fig. 2). This is particularly true for the $\mathrm{L}$ band, because both snow pack surface and volume component are increasing with the increase in frequency. The most important question is then how the snow density affects this component, which can be equalized with the whole backscattering coefficient, with the insignificant error.

\section{1. $L$ band}

In the $\mathrm{L}$ band, backscattering (underlying ground backscattering) is rising with the snow density increase. This phenomenon was noticed first time by Shi and Dozier [2] and can be explained by the fact that density affects backscattering dominantly through the change of air-snow interface refraction angle and the change of the wavelength in the snow medium. Snow density increase causes increase of snow dielectric constant, which defines electromagnetic waves phase velocity. Taking the relation between frequency and wavelength, it can be seen that increased dielectric constant, through decrease of waves phase velocity, causes wavelength shortening. In backscattering theory, smaller wavelength implies bigger apparent roughness and therefore stronger backscattering. According to Snell-Descartes law waves incident and refracted angle relation at the boundary between two different medium is given as:

$$
\frac{\sin \left(\theta_{i}\right)}{\sin \left(\theta_{r}\right)}=\sqrt{\frac{\varepsilon_{r}}{\varepsilon_{i}}}
$$

Snow cover is considered as refracted waves medium, implying that its dielectric constant increase leads to refracted angle decrease. Knowing that surface backscattering coefficient function against angle in $\mathrm{L}$ band has a negative slope, two previously described effects can justify the behaviour spotted in figure 3.

There is one more parameter potentially causing backscattering rise with density increase - extinction coefficient. By using two mentioned approaches in parallel, this possibility is declined. Namely, the backscattering curves for two models are approximately the same, which proves negligibility of the extinction and justifies figure.

Using this conclusion, increased sensitivity to the angle and wavelength change, Shi and Dozier created a model for deducing snow density from snow dielectric constant [2].

\section{2. $C$ band}

In the $\mathrm{C}$ band, however, for the same assumed roughness parameters, the previously mentioned effect is not present any more. Now, density increase causes decrease of backscattering (fig. 3).

Comparison between independent and multiple scattering approaches is again pointing to the extinction negligibility, Fresnel transmission coefficient is independent of frequency, which leaves only ground surface backscattering in the presence of snow behaviour change as the cause of the sensitivity direction change.

Snow density change can affect ground surface backscattering in the presence of snow by:

- changing ground incident waves wavelength and incident 

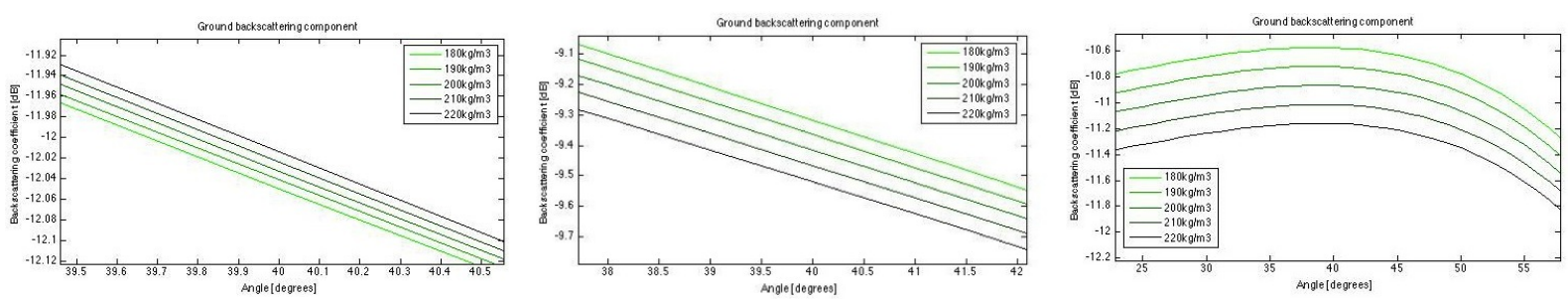

Fig. 3. Underlying ground backscattering component sensitivity on density change in $\mathrm{L}, \mathrm{C}$ and $\mathrm{X}$ band, VV polarization

angle at the ground surface;

- changing ground-snow dielectric contrast.

First effect, unlike it was in the case of L band, is not causing dramatic rise of backscattering. The explanation can be found in the fact that increase in frequency, supported by wavelength shortening leads to the surface backscattering angular distribution isotropy, which makes angle change to be almost insignificant for the backscattering. On the other side, ground-snow dielectric contrast decrease, which is the consequence of density increase, leads to the backscattering decrease (fig. 4).

\section{3. $X$ band}

In $\mathrm{X}$ band underlying ground component dominance is still maintained and the sensitivity direction remains the same as in C band, but significantly increased (fig. 3).

The justification is derived from ground surface backscattering in the presence of snow. Sensitivity related to the change of the dielectric contrast is independent of frequency, so it keeps the same direction and value as the one presented for C-band. On the other side, ground incident waves wavelength and incident angle change is having contra effect from the one characteristic for L band. Snow density increase causes backscattering decrease, due to the change of surface backscattering against angle function which slope becomes positive with frequency increase (fig. 4).
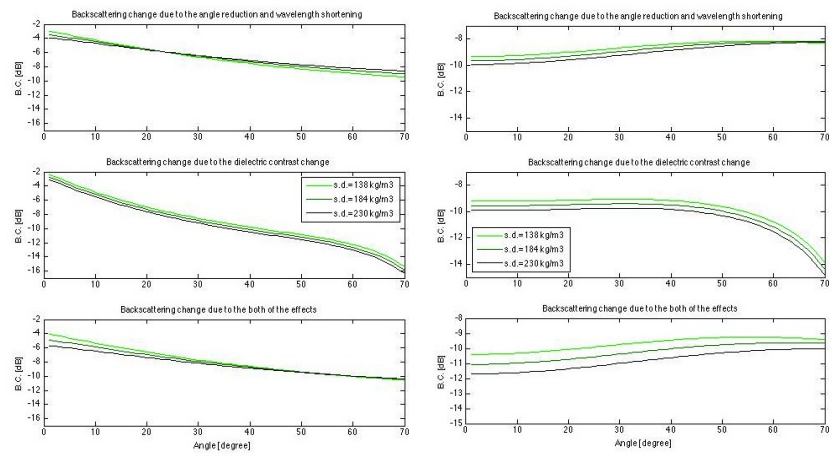

Fig. 4. $\sigma_{g r}$ in the presence of snow sensitivity on density change in $\mathrm{C}$ (left) and $\mathrm{X}$ (right) band, VV polarization

The introduced simulator validity is partly proven using the local snow density measurements conducted in Grand Rousse massif in French Alps at the dates (08.02.2009. and 19.02.2009.) corresponding to the images acquisition ones. Ratios between pixel intensities and simulator outputs $6.82 \cdot 10^{3}$ and $6.36 \cdot 10^{3}$ for HH channel, $4.33 \cdot 10^{3}$ and $4.11 \cdot 10^{3}$ for VV channel can be considered as the consistent ones, proving the reliability of the simulator.

\section{CONCLUSION}

The conclusion could be made that in $\mathrm{C}$ band, unlike the L [2], snow density dominantly affects total backscattering through the change of snow dielectric constant, which reflects to the change of ground-snow dielectric contrast. $\mathrm{X}$ band dry snow backscattering is characterized with the significant decrease of backscattering coefficient due to the density increase. Even the sensitivity is much bigger then the one found in $\mathrm{L}$ or $\mathrm{C}$ band, density impact is quite complex, because of approximately equal contribution of ground-snow dielectric contrast change and the change of ground incidence waves due to the presence of snow.

\section{REFERENCES}

[1] W. Gareth Rees, "Remote Sensing of Snow and Ice", CRC Press, Taylor and Franciss group, 2nd Edition, 2006.

[2] J. Shi, J. Dozier, "Estimation of Snow Water Equivalence Using SIR-C/X-SAR, Part I: Inferring Snow Density and Subsurface Properties", IEEE Transactions on Geosciences and Remote Sensing, vol.38, no.6, pp. 2465 - 2474, 2000.

[3] J. Shi, J. Dozier, "Estimation of Snow Water Equivalence Using SIR-C/X-SAR, Part II: Inferring Snow Depth and Particle Size", IEEE Transactions on Geosciences and Remote Sensing, vol.38, no.6, pp. 2475 - 2488, 2000.

[4] C. O. Ao, "Electromagnetic Wave Scattering by Discrete Random Media with Remote Sensing Applications", PhD Thesis, Massachusetts Institute of Technology, 2001.

[5] N. Longepe, S. Allain, L. Ferro-Famil, E. Pottier, Y. Durand "Snowpack Characterization in Mountainous Regions Using CBand SAR Data and a Meteorological Model", IEEE Transactions on Geosciences and Remote Sensing, vol.47, no.2, pp. 406418, 2009.

[6] A. K. Fung, D. Schutzer, K. S. Chen, "Microwave scattering and emission models for users", Artech House, 1nd Edition, 2010. 